\title{
Conceptual Foundation of the Fokker-Planck Approach to Space-Charge Effects
}

\author{
Courtlandt L. Bohn \\ Continuous Electron Beam Accelerator Facility ${ }^{1}$ \\ Newport News, VA 23606
}

\begin{abstract}
An rms-mismatched beam can evolve rapidly to a configuration of quasiequilibrium under the influence of space-charge forces. As it evolves, its emittance grows and a diffuse halo forms. The beam's distribution function accounts for all of the complicated dynamics. Unfortunately, the distribution function is difficult to calculate inasmuch as the physics lies at the interface between classical mechanics and thermodynamics. This paper presents the foundation for a statistical theory of the dynamics of nonequilibrium space-charge-dominated beams. Within certain approximations, the theory takes on a FokkerPlanck form. Key questions arise concerning the nature of the dynamical friction and diffusion in the beam's phase space and of the quasiequilibrium configuration that ensues.
\end{abstract}

\section{INTRODUCTION}

A charged-particle beam in an accelerator can be significantly away from thermal equilibrium. This is particularly true following transitions in the effective external focusing force. In an intense beam, space charge drives the subsequent evolution. These beams are generally "cool", and charge redistribution in response to the change in external focusing leads to wavebreaking in the beam's phase space, an event which marks the onset of turbulence. Excess free energy goes toward establishing a mode spectrum. The mode spectrum interacts with particle orbits to generate phase mixing, a process which generally decreases the coarse-grained phase-space density near the phase point of each particle. The fluctuating space-charge potential also widens the range of energies of the particles, a process known in the astrophysical community in connection with a fluctuating gravitational potential in a large stellar system as "violent relaxation".

Phase mixing and violent relaxation can drive the beam toward a quasiequilibrium state on a time scale much shorter than the (oulomb relaxation time. Several numerical simulations show relaxation if rm!s-mismatched

\footnotetext{
${ }^{1}$ CEBAF is operated by the Southeastern Universities Rese.arch Ansoriation for the Department of Energy under Contract DE-AC05-84ER40150
} 


\section{DISCLAIMER}

Portions of this document may be illegible in electronic image products. Images are produced from the best available original document. 
space-charge-dominated beams to quasiequilibrium within a few plasma periods, corresponding to only a few lattice periods (1). One might suspect this to be due to numerical noise. However, the process has also been unambiguously observed in laboratory experiments (2).

Inasmuch as they behave like nonneutral plasmas, three-dimensional beams which are strongly turbulent are characterized by an average "collision frequency" $\sim g^{-1}$ times larger than in a quiescent beam, where $g \equiv 1 / n \lambda_{D}^{3}$ is the plasma parameter (3), with $n$ representing the number density and $\lambda_{D}$ representing the Debye length. In space-charge-dominated beams $g^{-1}$ is large, and interactions between particles and localized fluctuations are important. In weak turbulence the average collision frequency is $\sim g^{1 / 2}$ times smaller than in strong turbulence.

Jean Delayen and I have recently advanced and applied a statistical theory of nonequilibrium beams $(4,5)$. It includes the evolving mode spectrum to account self-consistently for rapid changes in the electrostatic self-field of the beam. Readers interested in a detailed account are referred to these papers. The following section, which is largely extracted from Ref. (4), presents the fundamental conceptual foundation of the theory, outlining how an equation of the Fokker-Planck type can materialize from a "reduction" of Liouville's equation for the distribution function. Subsequent discussion presents key questions that arise in the context of this approach.

\section{FOKKER-PLANCK MODEL}

A precise statistical treatment of the beam dynamics involves the microscopic Klimontovich density distribution. This distribution consists of a selfconsistent superposition of the orbits of all constituent particles of the beam in the Cartesian phase space $(x, u)$ of a single particle, and it satisfies Liouville's theorem. The microscopic distribution is

$$
f(\mathbf{x}, \mathbf{u}, t)=\frac{1}{N} \sum_{i=1}^{N} \delta\left[\mathbf{x}-\mathbf{x}_{i}(t)\right] \delta\left[\mathbf{u}-\mathbf{u}_{i}(t)\right],
$$

where $\left[\mathbf{x}_{i}(t), \mathbf{u}_{i}(t)\right]$ denotes the orbit of the $i$ th particle, and $N$ is the total number of particles. The ultimate goal of a numerical experiment is to specify the Klimontovich distribution as a function of time.

To develop an analytic formalism, it is more practical to work with a macroscopic distribution function. We thus consider the macroscopic. coarse-grained distribution function $\bar{f}(\mathbf{x}, \mathbf{u}, t)$ which is found by averaging the Klimontovich distribution over scales substantially greater than those associated with localized turbulent fluctuations:

$$
\bar{f}(\mathbf{x}, \mathbf{u}, t)=\frac{1}{\Delta V(\mathbf{x}, \mathbf{u})} \int_{\Delta V(\mathbf{x}, \mathbf{u})} d \mathbf{x}^{\prime} d \mathbf{u}^{\prime} f\left(\mathbf{x}^{\prime}, \mathbf{u}^{\prime}, t\right) .
$$


where $\Delta V(\mathbf{x}, \mathbf{u})$ is a phase-space volume element centered on the coordinates $(x, u)$ which is large compared to the size of the turbulent fluctuations but small compared to the size of the beam. In what follows, the "bar" is a signature of quantities calculated from the coarse-grained distribution $\vec{f}$.

After resolving the Klimontovich distribution into two components, $\bar{f}$ and fluctuations about $\bar{f}$, and averaging Liouville's equation, we are left with a "collision" term involving the fluctuations. Working with a coarse-grained distribution function is tantamount to neglecting nonlinear coupling between fluctuations in the particle distribution and fluctuations in the electromagnetic field. This approach results in the reduction of Liouville's equation to an equation of the Fokker-Planck type (6):

$$
\left(\partial_{t}+\mathbf{u} \cdot \nabla_{x}+\overline{\mathbf{K}} \cdot \nabla_{u}\right) \bar{f}=\nabla_{u} \cdot(\mathbf{F} \bar{f})+\nabla_{u} \cdot\left(D \cdot \nabla_{u} \bar{f}\right),
$$

where $\overline{\mathbf{K}}$ is the net acceleration of a particle in the comoving frame found from the potentials $\Phi_{f}$ and $\bar{\Phi}_{s}$ associated with the external focusing force and coarse-grained internal space-charge force, respectively, i.e.,

$$
\overline{\mathbf{K}}=-Q M^{-1} \nabla_{x}\left(\Phi_{f}+\bar{\Phi}_{s}\right) ;
$$

the vector $F$ and tensor $D$ are coefficients of friction and diffusion, respectively, and $Q$ and $M$ are the charge and mass, respectively, of the beam particles. According to Poisson's equation, $\bar{\Phi}_{s}$ is determined from the coarse-grained density, which is in turn determined from $\bar{f}$ :

$$
\nabla_{x}^{2} \bar{\Phi}_{s}(\mathbf{x}, t)=-\frac{N Q}{\varepsilon_{0}} \int d u \bar{f}(\mathbf{x}, \mathbf{u}, t)
$$

in which $\varepsilon_{0}$ is the permittivity of free space.

If the coarse-grained beam is regarded to be uniform so that it can be Fourier transformed using the periodic boundary conditions of a homogeneous cube of volume $V$, then the transport coefficients are

$$
\mathbf{F}=\frac{1}{V} \frac{2 \pi Q^{2}}{M \varepsilon_{0}} \sum_{\mathbf{k}} \frac{\mathbf{k}}{k^{2}} \frac{\delta\left(\omega_{\mathbf{k}}-\mathbf{k} \cdot \mathbf{u}\right)}{\epsilon^{\prime}\left(\mathbf{k}, \omega_{\mathbf{k}}\right)}
$$

and

$$
\mathrm{D}=\frac{1}{V} \frac{2 \pi Q^{2}}{M^{2} \varepsilon_{0}} \sum_{\mathbf{k}} \frac{\mathcal{E}_{\mathbf{k}}}{\omega_{\mathbf{k}}} \frac{\mathbf{k} \mathbf{k}}{k^{2}} \frac{\delta\left(\omega_{\mathbf{k}}-\mathbf{k} \cdot \mathbf{u}\right)}{\epsilon^{\prime}\left(\mathbf{k}, \omega_{\mathbf{k}}\right)}
$$

In these expressions, $\mathcal{E}_{\mathbf{k}}$ is the energy contained $\|$ ih. Hutuation with

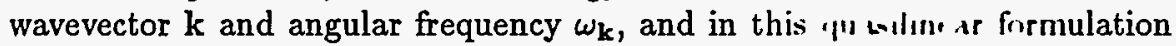
it evolves in the manner

$$
\frac{\partial \mathcal{E}_{\mathbf{k}}}{\partial t}=2 \gamma_{\mathbf{k}} \mathcal{E}_{\mathbf{k}}+\frac{\pi M \omega_{p}^{2} \omega_{\mathbf{k}}}{k^{2} \epsilon^{\prime}\left(\mathbf{k}, \omega_{\mathbf{k}}\right)} \int d \mathbf{u} \bar{f}(\mathbf{u}) \delta\left(\omega_{\mathbf{k}}-\mathbf{k} \quad \mathbf{1} \mathbf{\prime}\right.
$$


where

$$
\gamma_{\mathbf{k}} \simeq\left[\frac{\partial \Re[\epsilon(\mathbf{k}, \omega)]}{\partial \omega}\right]^{-1} \Im\left[\varepsilon\left(\mathbf{k}, \omega_{\mathbf{k}}\right)\right]
$$

The first term on the right-hand side of Eq. (8) accounts for Landau damping or growth at the rate $\gamma_{\mathbf{k}}$ from absorption or induced emission of mode energy by the particles, respectively, and the second term accounts for spontaneous Cherenkov emission of mode energy by the particles. The dielectric response function is

$$
\epsilon(\mathbf{k}, \omega)=1+\frac{\omega_{p}^{2}}{k^{2}} \int d \mathbf{u} \frac{\mathbf{k} \cdot \nabla_{u} \bar{f}(\mathbf{u})}{\omega-\mathbf{k} \cdot \mathbf{u}}
$$

$\epsilon^{\prime}\left(\mathbf{k}, \omega_{\mathbf{k}}\right)$ denotes $[\partial \epsilon / \partial \omega]_{\omega=\omega_{k}}, \omega=\omega_{\mathbf{k}}+i \gamma_{\mathbf{k}}$ is the solution of $\epsilon(\mathbf{k}, \omega)=0$, and $\omega_{p}^{2}=n Q^{2} / \varepsilon_{0} M$ is the square of the plasma frequency. The $\mathbf{k}$-summation in Eqs. (6) and (7) is over the growing (unstable) modes obtained from these zeroes, for which $\gamma_{\mathbf{k}}>0$. The friction $F$ arises from particles losing energy to unstable modes via spontaneous Cherenkov emission, and the diffusion $D$ results from particles recoiling in response to absorption and induced emission. In turn, diffusion gives rise to turbulent heating of the particles.

The effect of fluctuations is to change the shape of $\bar{f}$ continuously until there are no more growing modes and $\gamma_{\mathbf{k}}<0$ for all $\mathbf{k}$. When this has occurred, the modes quickly dissipate by linear Landau damping, and the turbulence vanishes. For example, in the presence of a background isotropic Maxwellian velocity distribution, the fluctuation spectrum evolves as

$$
\frac{\partial \mathcal{E}_{\mathbf{k}}}{\partial t}=\frac{\pi M \omega_{p}^{2} \omega_{\mathbf{k}}}{k^{2} \epsilon^{\prime}\left(\mathbf{k}, \omega_{\mathbf{k}}\right)}\left(1-\frac{\mathcal{E}_{\mathbf{k}}}{k_{B} T}\right) \int d \mathbf{u} \bar{f}(\mathbf{u}) \delta\left(\omega_{\mathbf{k}}-\mathbf{k} \cdot \mathbf{u}\right),
$$

in which $k_{B}$ is Boltzmann's constant and $T$ is the beam's thermal-equilibrium temperature. This shows explicitly that $\mathcal{E}_{\mathrm{k}}$ dissipates if $\mathcal{E}_{\mathrm{k}}>k_{B} T$, and the energy spectrum thermalizes toward the equipartition value $\mathcal{E}_{\mathrm{k}}=k_{B} T$.

In the Fokker-Planck equation (3), the left-hand side accounts for systematic effects arising from the external focusing field and the coarse-grained space-charge field, and it therefore includes resonances between global spacecharge modes and the focusing force if any are present. The process of phase mixing is included there. The right-hand side accounts for stochastic effects of the collective-mode spectrum. Fast collisionless relaxation and energy redistribution between modes and particles are included there.

This quasilinear formalism highlights the ingredients of a self-consistent solution for the dynamics of a nonequilibrium beam. As the fluct uations evolve, they change the shape of the coarse-grained distribution function, which in turn modifies the evolution of the fluctuations. As the mode spectrum dissipates, the friction and diffusion coefficients eventually settle down toward their thermal values associated with Coulomb collisions. and the beam then 
slowly proceeds to thermal equilibrium. A word of caution, however: with respect to charged-particle beams, "thermal equilibrium" has meaning only in the approximation that the frequency of the external focusing forces is high enough that there is no significant beam evolution between focusing lenses or acceleration gaps.

\section{KEY QUESTIONS}

The statistical theory is obviously very complicated. It is also only approximate, particularly with regard to its application to an inhomogeneous beam, for in this case it is very difficult to calculate the normal modes. Yet, at the same time, it simplifies matters by operating over the 6-dimensional phase space of a single particle rather than the $6 \mathrm{~N}$-dimensional phase space of the $N$ particles comprising a beam bunch.

To date, applications have incorporated a simple model of the turbulence and Brownian particle motion to specify the Fokker-Planck coefficients. Practical applications of the statistical theory therefore require more accurate models of these coefficients. Establishing viable models will undoubtedly require carefully planned numerical experiments. A fundamental approach would be to calculate the fluctuation spectrum at each position and time step during the course of a numerical experiment and then construct the coefficients from the spectrum using Eqs. (6) and (7) as guides. A second approach might be to infer them from the orbits of test particles in a sequence of numerical experiments. It may also prove fruitful to try to measure these quantities in laboratory beams.

Mismatch-induced excitation and subsequent relaxation of a turbulent mode spectrum constitutes a transient phase in the beam's evolution. As the fluctuations damp, the beam will evolve more slowly. It is not obvious that violent relaxation drives the beam all the way to thermodynamic equilibrium. The process stops when the potential ceases to change and there is no guarantee that the excess free energy is fully equilibrated at that time.

Numerical simulations and laboratory experiments suggest the beam assumes a configuration of quasiequilibrium after a few plasma periods. At this stage the microscopic fluctuations are probably weak, and they will eventually damp to thermal levels. Subsequent evolution is very slow and, except in circular machines, will take much longer than the transit time of the beam through the remainder of the accelerator. Thus, it is important to establish the properties of the quasiequilibrium beam, especially in machines where there is concern about radioactivation due to scraping of a diffuse halo. For example, if the quasiequilibrium beam were to comprise only a few low-order global modes of oscillation, then models describing the oscillating-core-singleparticle interaction (7) would probably be sufficient to compute the bulk of the halo over most of the machine. One would need to be careful, though, because in the early transient stage a statistically few particles could conceiv- 
ably interact resonantly with many modes and be launched into orbits of very large amplitude. This is a consideration, for example, in high-current proton machines envisioned as spallation neutron sources, for which losses less than a $\mathrm{nA} / \mathrm{m}$ are of concern (8).

One possibly fruitful approach might be to formulate a "maximum-entropy" principle. It would take into account that not all "final" states are equally probable in the configuration of quasiequilibrium. For example, there is not enough time available to populate the largest-amplitude orbits in the MaxwellBoltzmann distribution. Accordingly, one would expect the core to have progressed closer to thermal equilibrium than the halo, and this is consistent with observations from laboratory and numerical experiments. This approach has been applied to self-gravitating stellar systems with some success (9).

A third area of investigation of possible relevance to accelerators is to consider explicitly a periodic driving force in a frame comoving with the beam. Stochastic processes in the presence of periodic forcing may be susceptible to resonances, which in turn will influence the dynamics. This has been seen, for example, in bistable systems (10). It is also of interest to establish unambiguously the properties of a beam in thermal equilibrium subject to a periodic external force.

\section{REFERENCES}

1. See, for example, T. P. Wangler, in High-Brightness Beams for Advanced Accelerator Applications, edited by W. W. Destler and S. K. Guharay, AIP Conf. Proc. 253, (AIP, New York, 1992), pp. 47-56; R. A. Jameson, Los Alamos Report No. LA-UR-93- 12, 1993 (unpublished), and also related articles in this Proceedings.

2. D. Kehne, M. Reiser, H. Rudd, in High-Brightness Beams for Advanced Accelerator Applications, ibid., pp. 47-56, and related discussion in these Proceedings.

3. S. Ichimaru, Basic Principles of Plasma Physics (Benjamin, Reading, MA, 1973), Chap. 11.

4. For a comprehensive account, see C. L. Bohn and J. R. Delayen, Phys. Rev. E 50, 1516 (1990).

5. The theory was introduced in C. L. Bohn, Phys. Rev. Lett. 70. 932 (1990), and it has been applied in C. L. Bohn and J. R. Delayen. Proc. 1994 Linac Conf., KEK Report, K. Takata, ed., 505 (1995), and in International ronference on Accelerator-Driven Transmutation Technologies and Applicatsons, edited by E. D. Arthur, A. Rodriguez, and S. O. Schriber, AIP Conf. Pror. 346. pp. 371-375 (1995).

6. S. Ichimaru's textbooks are good starting references: Ref $(1)$ and Statistical Plasma Physics, Vol. 1 (Addison-Wesley, Redwood (its. ( 1.1192$)$. The quasilinear formalism described herein is based largely on hu triatment. D. Pesme, Phys. Scr. T50, 7 (1994) argues that the Fokker-Planck eylustion may also apply to systems in which nonlinear coupling predominates. provided the transport coefficients are judiciously chosen. 
7. See, for example, R. L. Gluckstern, Phys. Rev. Lett. 73, 1247 (1994), and related articles in this Proceedings.

8. J. R. Delayen and C. L. Bohn, in International Conference on Accelerator-Driven Transmutation Technologies and Applications, op. cit., pp. 460-465.

9. D. N. Spergel and L. Hernquist, Astrophys. J. Lett. 397, L75 (1992).

10. P. Jung, Phys. Reports 234, 175 (1993). 